\title{
Comunidades fungicas asociadas a sustratos leñosos en un rio mediterraneo
}

\author{
A. Roldán 1 \\ Mạ Angeles Puig²
}

M. Honrubial

Palabras clave : Hifomicetos acuáticos, madera, río Vinalopó, España.

Se describe una experiencia llevada a cabo en la cabecera del rio Vinalopó (Alicante, España). Con el empleo de bloques de madera de chopo como « cebos " se determinaron las pautas de sucesión de la flora fúngica saprófita. Trece especies de hifomicetos intervienen en los procesos de degradación de la madera. En medio léntico el número de hifomicetos presentes en los bloques es siempre menor que en la zona de corriente. Los valores maximos de diversidad fúngica se alcazan antes en medio léntico. En medio lótico se observa un proceso de sucesión en el que Cylindrocarpon ianthothele Wollenw. y Heliscus lugdunensis Sacc. \& Therry son progresivamente reemplazados por otras especies.

Fungal communities associated with wood test-blocks in a mediterranean stream.

Keywords : Aquatic Hyphomycetes, wood, Vinalopó river, Spain.

Fungal communities in the river Vinalopó (Alicante, Spain) have been surveyed with wood test-blocks. Thirteen species of aquatic hyphomycetes were detected in this study. The number of hyphomycetes is always greater in a lentic habitat than in lotic one. Highest values of fungal diversity are obtained earlier in lentic habitat. In lotic parts of the river some sucessional patterns have been observed : Cylindrocarpon ianthothele Wollenw. and Heliscus lugdunensis Sacc. \& Therry were early colonizers and their presence decreased progressively.

\section{Introducción}

Los residuos de madera y otros restos vegetales muy lignificados, como estróbilos y aciculas de coníferas, tienen un carácter menos estacional y sus procesos degradativos son mucho más lentos que los de las hojas, consecuentemente proveen una fuente de sustrato estable para los microorganismos descomponedores (Willoughby \& Archer 1973). Al mismo tiempo, la larga permanencia de estos sustratos en el cauce permite estudiar con mayor facilidad fenómenos de sucesión en su flora fúngica saprófita.

En la literatura mundial encontramos gran número de trabajos sobre los aspectos florísticos y ecológicos de la descomposición de madera en medio

1. Depto. Biologia Vegetal. Facultad Biologia. Universidad de Murcia. Campus de Espinardo 30071 Murcia. España.

2. Depto. Biologia Animal y Ecologia. Facultad Biologia. Universidad de Murcia. Campus de Espinardo 30071 Murcia. España. marino (Kohlmeyer \& Kohlmeyer 1979) o en estuaios (Kirk 1969) ; pero son más escasos los que se centran en cursos de agua dulce (Willougnby 1971 ; Willoughby \& Archer 1973, Shearer 1972, Sanders \& Anderson 1979, Lamore \& Goos 1978), y ninguno de ellos en ríos mediterráneos o sistemas equiparables al río Vinalopó.

\section{Material y métodos}

El estudio de la flora fúngica y sus pautas de sucesión en madera presenta serias dificultades, ya que la identificación de los hifomicetos acuáticos requiere el transporte del material al laboratorio y, con esta metodología, se interfiere en gran medida con los procesos naturales. Con el fin de minimizar estas interferencias se prepararon series de « cebos» con bloques de madera de chopo con medidas estandarizadas $(8 \times 5 \times 3 \mathrm{~cm})$ previamente esterilizados y sometidos en estufa a $120^{\circ} \mathrm{C}$ durante 24 horas para determinar su peso seco. Paquetes con cinco de estos bloques se colocaron, respectivemente, 
respectivamente, en zonas de la cabecera del río muy próximas entre sí. Una de ellas situada en un tramo de corriente y la segunda en un remanso con corriente prácticamente nula.

Los distintos bloques se recogieron individual y sucesivamente $\mathrm{y}$, de este modo, se extrapolan los resultados como si en cada zona el bloque estudiado fuera único. En el laboratorio, los bloques se cometieron a cultivo en aireación durante 48 horas, según la metodología detallada en Roldan et al. (1987), y con las suspensiones de conidios obtenidas se elaboraron los porcentajes relativos de presencia para cada especie. En trabajos similares llevados a cabo por otros autores (vgr. Willoughby \& Archer 1973, Lamore \& Goos 1978) el procedimiento seguido difiere del aquí empleado : una vez recolectados los cebos, se disponen en cámara húmeda durante periodos de tiempo que oscilan desde dos semanas hasta más de un ano. Con este tratamiento se consigue un amplio catálogo de hongos de los más diversos grupos, pero se ha estimado que no corresponde al estado presumible de la flora fúngica in situ. Largos periodos de incubación en cámara húmeda favorecen la proliferación de especies que en el río pueden estar muy mermadas en sus facultades descomponedoras, en perjuicio de las estrictamente acuáticas con verdadera importancia en su medio natural.

Las pérdidas en peso seco del material estudiado se obtuvieron por diferencia tras someter los bloques de madera a una temperatura de $120^{\circ} \mathrm{C}$ durante 24 horas.

El estudio se llevó a cabo en la cabecera del río Vinalopó, en una zona caliza situada en el límite de las provincias de Alicante y Valencia (S.E. de España), con una altitud de $800 \mathrm{~ms}$. En la tabla 1 se exponen los datos correspondientes a los análisis físico-químicos realizados paralelamente a la puesta y recogida de los cebos.

\section{Resultados y discusion}

En el transcurso de la experiencia se han reconocido 13 taxones fúngicos (ver tabla 2), uno de ellos sin posible identificación pero muy afin a Dimorphospora foliicola Tubaki. Todas las especies, con excepción de Trichocladium angelicum Roldán \& Honrubia y la forma desconocida ya mencionada, se han encontrado colonizando hojas y también en muestras de espuma de la misma localidad. Estos dos taxones parecen ser especificos de madera en el
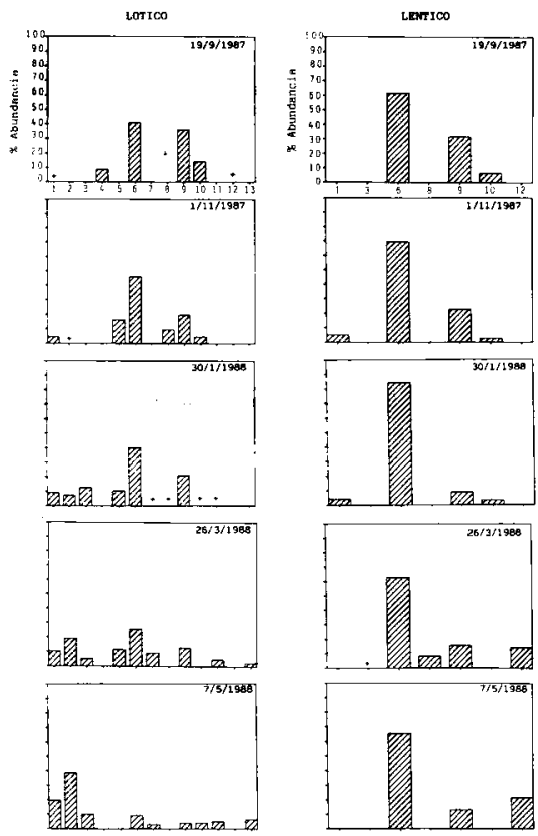

Fig. 1. Porcentajes de abundancia relativa de las especies fúngicas en cada muestreo. Las cifras en horizontal representan las especies de hongos con la numeración seguida en la Tabla 2.

río Vinalopó. Willoughby \& Archer (1973) encontraron una flora fúngica sobre madera menos numerosa que en muestras de espuma de la misma localidad, con varios taxones específicos para este sustrato. Incluso Jones \& Oliver (1964) apuntan hacia un posible preferencia de algunos hifomicetos por algún tipo de madera determinado.

Volucrispora graminea Ingold, Mc Dougall \& Dann es la primera vez que se cita sobre madera, aunque las peculiaridades morfológicas de las cepas aisladas en cultivo puro no descartan tajantemente que pueda tratarse de otra especie o variedad.

En la fig. 1 se ilustran los porcentajes de las distintas especies en cada muestreo y subestación 
Tabla 1. Valores numéricos de los parámetros físico-químicos en el río Vinalopó paralejamente a la colocación y recogida de los " cebos ".

\begin{tabular}{|c|c|c|c|c|c|c|c|c|c|}
\hline & Fecha & $\mathrm{T}^{\circ} \mathrm{C}$ & $\mathrm{pH}$ & $\begin{array}{c}\text { Oxigeno } \\
\text { (ppm) }\end{array}$ & $\begin{array}{c}\text { Cond. } \\
\left({ }_{\mu} s\right)\end{array}$ & $\begin{array}{l}\text { Sol. susp. } \\
\text { (mg/l) }\end{array}$ & $\begin{array}{c}\text { Fosfatos } \\
(\mu \mathrm{g}, \mathrm{atm} / \mathrm{l})\end{array}$ & $\begin{array}{l}\text { Nitritos } \\
(\mu \mathrm{g}, \mathrm{atm} / \mathrm{l})\end{array}$ & $\begin{array}{c}\text { Amonio } \\
\text { (ppm) }\end{array}$ \\
\hline \multicolumn{10}{|l|}{ LENTICO } \\
\hline Puesta & $6 / 9 / 87$ & 14.3 & 8.3 & 2.8 & 260 & 75 & 30.1 & 0.17 & 0.950 \\
\hline 1] recogida & $19 / 9 / 87$ & 15 & 8.3 & 5.2 & 410 & 55 & 21.7 & 0.35 & 0.573 \\
\hline 3a recogida & $1 / 11 / 87$ & 13.2 & 8.8 & 5.3 & 140 & 90 & 23.8 & 0.25 & 0.807 \\
\hline 3a recogida & $30 / 1 / 88$ & 11.8 & 7.7 & 4.8 & 150 & 21 & 21.5 & 0.35 & 0.931 \\
\hline 4â recogida & $26 / 3 / 88$ & 10.8 & 8.4 & 7.1 & 220 & 15 & 34 & 0.54 & 0.005 \\
\hline $5 \underline{a}$ recogida & $7 / 5 / 88$ & 16.5 & 8.0 & 4.1 & 250 & 43 & 18.7 & 1.33 & 0.230 \\
\hline \multicolumn{10}{|l|}{ LOTICO } \\
\hline Puesta & $6 / 9 / 87$ & 15.1 & 8.2 & 8.3 & 260 & 105 & 26.9 & 0.25 & 0.035 \\
\hline lạ recogida & $19 / 9 / 87$ & 15 & 7.9 & 9.5 & 250 & 52 & 23.3 & 0.35 & 0.453 \\
\hline 2a recogida & $1 / 11 / 87$ & 14.1 & 8.8 & 8.9 & 130 & 60 & 25.4 & 0.35 & 0.003 \\
\hline 3a recogida & $30 / 1 / 88$ & 13.2 & 7.5 & 9.8 & 140 & 4 & 27.8 & 0.54 & 0.039 \\
\hline 4ạ recogida & $26 / 3 / 88$ & 13.2 & 8.3 & 9.5 & 160 & 5 & 20.7 & 0.74 & 0.639 \\
\hline 5a recogida & $7 / 5 / 88$ & 15.1 & 7.4 & 8.6 & 190 & 37 & 25.6 & 1.53 & 0.045 \\
\hline
\end{tabular}

Tabla 2. - Frecuencias de aparición de cada especie en todas las campañas.

\begin{tabular}{|c|c|c|c|c|c|c|c|c|c|c|}
\hline & \multicolumn{5}{|c|}{ LOTICO } & \multicolumn{5}{|c|}{ LENTICO } \\
\hline & $19 / 9 / 87$ & $1 / 11 / 87$ & $30 / 1 / 88$ & $26 / 3 / 88$ & $7 / 5 / 88$ & $19 / 9 / 87$ & $1 / 11 / 87$ & $30 / 1 / 86$ & $2653 / 88$ & $7 / 5 / 88$ \\
\hline 1 Alotospora acuminata Ingold & 0.01 & 4.36 & 9.25 & 10.36 & 19.42 & & 5.21 & 4.10 & & \\
\hline 2 Alatospora pulchella Marvanová & & 0.01 & 7.14 & 18.74 & 38.75 & & & & & \\
\hline $\begin{array}{l}3 \text { Anguillospora longissima (Sacc. \& } \\
\text { Sydow) Ingold }\end{array}$ & & & 12.56 & 5.02 & 10.10 & & & & 0.01 & \\
\hline 4 Articulospora antipodes nom. ined & 8.76 & & & & & & & & & \\
\hline 5 Clavariopsis aquatica De Wild. & & 16.15 & 10.20 & 11.47 & & & & & & \\
\hline 6 Cylindrocarpon ianthothele & & & & & & & & & & \\
\hline Woljenw. & 41.15 & 46.12 & 39.89 & 25.54 & 9.21 & 61.83 & 69.42 & 84.12 & 62.41 & 65.56 \\
\hline 7 Flagellosporo penicillioides Ingold & & & 0.01 & 9.25 & 2.87 & & & & & \\
\hline 8 Fusarium culmorum (Smith) Sacc. & & 9.32 & 0.01 & & & & & & 8.13 & \\
\hline 9 Heliscus lugdunensis Sacc. \& Therry & 36.22 & 19.60 & 20.93 & 12.76 & 4.12 & 31.15 & 22.73 & 8.74 & 15.24 & 12.97 \\
\hline $\begin{array}{l}10 \text { Tetraciadium marchalianum De } \\
\text { Wild }\end{array}$ & 13.85 & 4.45 & 0.01 & & 4.10 & 6.42 & 2.64 & 3.04 & & \\
\hline $\begin{array}{l}11 \text { Trichociadium angelicum Roldán \& } \\
\text { Honrubia }\end{array}$ & & & 0.01 & 4.36 & 5.25 & & & & & \\
\hline $\begin{array}{l}12 \text { Volucrispora graminea Ingold, Mc } \\
\text { Dougall \& Dann }\end{array}$ & 0.01 & & & & & & & & 14.21 & 21.47 \\
\hline 13 Desconocido & & & & 2.50 & 6.18 & & & & & \\
\hline
\end{tabular}


considerada. La zona léntica, confirmando los resultados que en experiencias paralelas se poseen para hojas, soporta un menor número de especies (ocho en total), que la lótica. Esta disminución en el número de especies en la zona léntica puede atribuirse al factor corriente, ya que la físico-química de ambas subestaciones es muy similar. Sólo se diferencian en los valores de oxigeno disuelto, que son menores en la zona embalsada pero nunca se acercan a los límites de la anoxia. La evolución sucesional de la flora fúngica es bastante pareja en ambas zonas, al menos en los primeros muestreos. Predominan, en este primer estadio, dos especies de hifomicetos con teleomorfo conocido en los pirenomicetos : Cylindrocarpon ianthothele y Heliscus lugdunensis. Acompanados con frecuencia bastante inferior por Tetracladium marchalianum De Wild. y Articulospora antipodes nom. ined., esta última exclusiva de corriente. En la zona léntica, las dos especies mencionadas en primer lugar son el componente mayoritario durante todo el ciclo, posteriormente se les agregan Alatospora acuminala Ingold $\mathrm{y}$, en los últimos muestreos, Volucrispora graminea en detrimento, sobre todo, de las frecuencias de Heliscus lugdunensis.

En la zona lótica la flora fúngica es mucho más diversa y se observa un proceso de sucesion en el que $C$. ianthothele y $H$. lugdunensis son progresivamnente reemplazados por otras especies, algunas de ellas siguen la pauta general temporal ya observada sobre hojas, como Alatospora pulchella Marv. y Clavariopsis aquatica De Wild. Otras, como es el caso de Flagellospora penicillioides Ingold y Fusarium culmorum (Smith) Sacc., no se ajustan al ciclo general de las mismas en la estación y muestran unas pautas independientes. Los restantes hifomicetos que aparecen son Trichocladium angelicum y una forma desconocida. En condiciones de cultivo, el crecimiento de estas dos especies es extraordinariamente lento. Quizá por este motivo sólo se presentan en sustratos con largo tiempo de permanencia en condiciones de inmersión.

En ambos ambientes, la pauta general observada es la presencia de especies pioneras ( $C$. ianthothete y Heliscus (ugdunensis) que colonizan rápidamente estos sustratos, aunque después siguen unas directrices diferentes que dependen del factor corriente. Esta tendencia general ya ha sido observada por Willoughby \& Archer (1973). En sus experimentos, Fusarium ssp. y Heliscus lugdunensis son las primeras especies que colonizaron sus maderas, incluso a las dos semanas de permanencia en el cauce. La diferencia con los datos obtenidos en el río Vinalopó estriba en que el papel de Fusarium ssp. es llevado a cabo por Cylindrocarpon ianthothele. En la experiencia de los autores citados, las especies pioneras conservaron sus frecuencias durante un ticmpo aproximado de cuatro meses, para después descender paulatinamente. En nuestro caso coincide con lo observado en la zona léntica pero no en la lótica, donde los fenómenos de sucesión son mucho más rápidos y activos.

Parece ser que el carácter de primer colonizador de una especie viene determinado por las caracteristicas intrinsecas del río. En experiencias parecidas efectuadas en Rhode Island (USA), Lamore \& Goos (1978) encontraron que Anguillospora longissima (Sacc. \& Sydow) Ingold tenía gran importancia cuantitativa en los primeros muestreos (en el rio Vinalopó aparece a los tres meses de permanencia del sustrato en condiciones de inmersión) y, sin embargo, Heliscus lugdunensis se definió como un hongo de tardía aparición.

Con respecto a Jas tasas de pérdida en peso seco de los bloques de madera, en las dos primeras semanas de permanencia en el agua se observa una notable pérdida en ambas subestaciones, presumiblemente por dilución de elementos solubes. Para comprobar esta hipótesis se sometieron varios testigos a inmersión con agua estéril en el laboratorio. Las pérdidas en peso observadas en este caso oscilaron entre el 1 y $1,5 \%$, equiparables a las primeras pérdidas de la experiencia. Tras este primer periodo la tendencia se dirige hacia una estabilización en los meses invernales. Siempre los bloques de madera situados en la zona de corriente sufren unas pérdidas mayores. En el último muestreo, el ascenso de las temperaturas provoca un apreciable incremento en la zona lótica, que se corresponde con un descenso de dificil interpretación en la subestación léntica.

Si se consideran las relaciones entre los porcentajes de pérdida relativos y la diversidad del componente füngico (fig. 2 y 3 ), se observa que las tendencias de la diversidad acumulada (indice de Shanon-Weber) oscilan poco entre ambas zonas. En léntico los valores se sitúan siempre por debajo de 1.5 bits y en lótico oscilan entre 2.5 y 3 bits. Los valores de diversidad, de algún modo indicativos del 


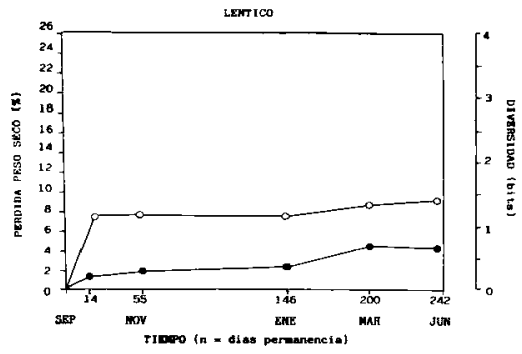

Fig. 2. - Gráficas de variación de los porcentajes de pérdida relativa en peso seco $(\bullet)$ y del índice de diversidad de ShanonWeber para al componente fúngico (o). Zona léntica.

grado de evolución de la comunidad, se estabilizan en la zona de corriente a los 2-4 meses de la permanencia de los cebos en el agua, aunque la tendencia hacia ligeros aumentos continúa hasta marzo. En la zona embalsada estos valores oscilan muy poco desde el primer muestreo. En la subestación léntica, las curvás de diversidad acumulada y pérdida de peso son muy parejas ; por el contrario, en la zona de corriente la mayor pérdida de peso que se observa al principio de la primavera no se corresponde con un aumento de la diversidad.

\section{Travaux cités}

Jones (E.B.G.) \& Oliver (A.C.). 1964. - Occurence of aquatic Hyphomycetes on wood submerged in fresh and brackish water. Trans. Br. mycol. Soc. $47: 45-48$.

Kirk (P.). 1969. - Aquatic hyphomycetes on wood in a estuary. Mycologia 61 : 177-181.

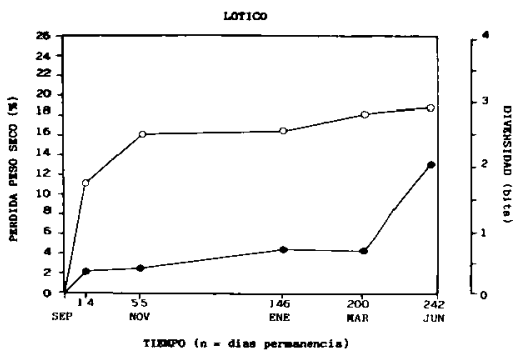

Fig. 3. - Gráficas de variación de los porcentajes de pérdida relativa en peso seco $(\bullet)$ y del indice de diversidad de ShanonWeber para el componente fúngico (o). Zona lótica.

Kohlmeyer (J.) \& Kohlmeyer (E.). 1979 : Marine Mycology. The Higher Fungi, Academic Press. New York.

Lamore (B.J.) \& Goos (R.D.). 1978. - Wood-inhabiting fungi of a freshwater stream in Rhode Island. Mycologia 70: 1025-1034.

Roldan (A.), Descals (E.) \& Honrubia (M.). 1987. - Notas sobre hifomicetos acuáticos saprfitos en restos vegetales. Cryptogamie, Mycol. 8(1): 61-66.

Sanders (P.F.) \& Anderson (J.M.). 1979. - Colonization of wood blocks by aquatic hyphomycetes. Trans. Br. mycol. Soc. $73: 103-107$.

Shearer (C.A.). 1972. - Fungi of the Chesapeake Bay and its tributaries. III. The distribution of wood-inhabiting Ascomycetes and Fungi Imperfecti of the Patuxent River. Amer. $J$. Bot. 59 : $961-969$.

Willoughby (L.G.). 1971. - Observations on some aquatic Actinomycetes of streams and rivers. Freshwater Biol. 1 : 23-27.

Willoughby (L.G.) \& Archer (J.F.). 1973. - The fungal flora of a freshwater stream and its colonization pattern on wood. Freshwater Biol, 3 : 219-239. 